\title{
Publisher's Note: Spreading law of non-Newtonian power-law liquids on a spherical substrate by an energy-balance approach [Phys. Rev. E 96, 012803 (2017)]
}

\author{
Masao Iwamatsu \\ (Received 6 October 2017; published 12 October 2017)
}

DOI: 10.1103/PhysRevE.96.049902

This paper was published online on 24 July 2017 with errors in Eqs. (26), (27), and (31), as well as the text following Eq. (31). Equation (26) should read as

$$
r_{\mathrm{L}}=R \sin \phi \simeq R \psi \simeq \frac{r_{0}}{r_{0}-R} R \theta
$$

Equation (27) should read as

$$
\theta^{2 n-1}\left[\frac{1}{2}\left(\theta^{2}-\theta_{\mathrm{Y}}^{2}\right)+\frac{r_{0}-R}{r_{0} \theta} \tilde{\tau}\right]=\lambda\left(\frac{2 n+1}{n}\right)^{n}\left(\frac{r_{0}}{r_{0}-R} R\right)^{1-n} \frac{\kappa U^{n}}{\sigma_{\mathrm{LV}}}
$$

Equation (31) should read as

$$
\Gamma=\left[\lambda\left(\frac{r_{0}}{r_{0}-R}\right) \frac{\kappa}{\sigma_{\mathrm{LV}}}\right]^{\frac{1}{n}}\left(\frac{2 n+1}{n}\right) R^{\frac{1}{n}} .
$$

The first two sentences following Eq. (31) should read as "Note that this coefficient $\Gamma$ depends on the power exponent $n$, the radius of the substrate $R$ and the volume of the droplet $V_{0}$ through $r_{0}$, and, in particular, $\Gamma$ is proportional to the $(1 / n)$ th power of the radius $R$ of the substrate $\Gamma \propto R^{1 / n}$. When $n=1$, Eq. (30) reduces to the equation for Newtonian liquids [26].” The paper has been corrected as of 6 October 2017. The equations and text are incorrect in the printed version of the journal. 